\title{
Implementación de la guía de buenas prácticas: valoración del riesgo y prevención de úlceras por presión: experiencia en la Fundación Oftalmológica de Santander (FOSCAL)
}

\author{
Implementation of the Good Practices Guide: Risk Assessment and \\ Prevention of Pressure Ulcers, an Experience at "Fundacion \\ Oftalmológica of Santander" (FOSCAL)
}

\section{Implementando do manual de boas práticas: avaliação do risco e prevenção de úlceras de pressão: experiênciana Fundação Oftalmológica do Santander (FOSCAL)}

\author{
Maribel Esparza-Bohórquez, Enf. * \\ Lina María Granados-Oliveros, Enf. ** \\ Katherinne Joya-Guevara, Enf. ***
}

\begin{abstract}
Resumen
Introducción: Desde hace tiempo se reconoce la importancia de la valoración del riesgo como estrategia de prevención del desarrollo de úlceras por presión (UPP) existen guías de práctica clínica que se caracterizan por recolectar la mejor evidencia disponible para este tipo de recomendación, entre ellas se encuentra la guía de valoración del riesgo y prevención de úlceras por presión de la Registered Nurses Association of Ontario (RNAO). Las úlceras por presión son un problema durante el proceso de atención hospitalaria porque afectan y deterioran el estado de salud, la calidad de vida y la funcionalidad familiar, sin olvidar las repercusiones económicas y legales para las instituciones. Objetivo: Describir la experiencia en la implementación de la guía de buenas prácticas de enfermería de la RNAO en dos servicios de hospitalización y en la unidad de cuidado intensivo adulto, durante un periodo
\end{abstract}

entre 2013 y 2015. Metodología: Estudio descriptivo prospectivo de implementación de la guía de valoración del riesgo y prevención de úlceras por presión de la RNAO, en dos servicios de hospitalización y en la unidad de cuidado intensivo adulto, organizado en dos fases: a) Fase Preparación: convenio, selección y capacitación; b) Fase Implementación: auditoría y seguimiento. Resultados: Para la guía de valoración del riesgo y prevención de úlceras por presión se trabajó con una población de 16,264 usuarios hospitalizados, de los cuales $6,712(41 \%)$ se clasificaron con riesgo de desarrollar UPP al ingreso de la hospitalización; se les practicó intervención de superficies de apoyo a 5,196 ( $77 \%$ ) y un total de 250 (4\%) pacientes desarrollaron UPP en estadio 2, evidenciando anualmente disminución gradual en la prevalencia. La adherencia del personal de enfermería al cumplimiento de las recomendaciones de la guía fue del 91\%. Conclusiones: Se evidenció en el ingreso de los pacientes al servicio, un aumento en el porcentaje de

* Enfermera, Jefe División de Enfermería, Fundación Oftalmológica de Santander (FOSCAL), Floridablanca, Santander, Colombia ** Coordinadora de Hospitalización, Fundación Oftalmológica de Santander (FOSCAL), Floridablanca, Santander, Colombia.

${ }^{* * *}$ Coordinadora del Estándar Hospitalario, Fundación Oftalmológica de Santander (FOSCAL), Floridablanca, Santander, Colombia

Autor de Correspondencia: Lina María Granados Oliveros, Coordinadora de Hospitalización, Fundación Oftalmológica de Santander FOSCAL, Torre Milton Salazar, piso 3, Urbanización El Bosque, Floridablanca, Santander, Colombia. Correo electrónico: coordinadora.hospitalizacion@foscal.com.co 
valoración del riesgo, seguimiento y revaloración durante la hospitalización. [Esparza-Bohórquez M, Granados-Oliveros LM, Joya-Guevara K. Implementación de la guía de buenas prácticas: valoración del riesgo y prevención de úlceras por presión: experiencia en la Fundación Oftalmológica de Santander (FOSCAL). MedUNAB 2016; 19(2):115-123].

Palabras clave: Úlcera por presión; Guía de práctica clínica; Cuidados de la piel; Enfermería; Riesgo.

\section{Abstract}

Introduction: For a long time, the importance of risk assessment as a strategy to prevent the development of pressure ulcers (PU) has been recognized, that is why there are clinical practice guidelines that are characterized by collecting the best available evidence for this type of recommendation, among them can be found the risk assessment and pressure ulcer prevention guidelines from the Registered Nurses Association of Ontario (RNAO). Pressure ulcers are a problem during the hospital care process because they affect and deteriorate the state of health, quality of life and family functionality, without forgetting the economic and legal repercussions for medical institutions. Objective: To describe the experience in the implementation of the RNAO nursing best practice guidelines in two hospitalization services, and another one with an adult in the intensive care unit from 2013 to 2015. Methodology: A prospective descriptive study was carried out about the implementation of risk assessment and prevention of pressure ulcers guidelines by RNAO in two hospitalization services, and another one with an adult in the intensive care unit which was organized in two phases a) Preparation phase: agreement, selection and training; b) Implementation phase: audit and follow-up. Results: For the risk assessment and prevention of pressure ulcer guidelines, a population of 16,264 inpatients were assessed, from which $6,712(41 \%)$ were classified, during their admission, as having a risk to develop PU; $5,196(77 \%)$ had a supportive surface area, and a total of $250(4 \%)$ patients developed stage $2 \mathrm{PU}$, showing a gradual decreasing rate in prevalence per year. The staff compliance adherence with the recommendations of the guidelines was $91 \%$. Conclusions: There was an increasing rate in the percentage of risk assessment, follow-up, and reassessment during hospitalization. [Esparza-Bohórquez M, Granados-Oliveros LM, Joya-Guevara K. Implementation of the Good Practices Guide: Risk Assessment and Prevention of Pressure Ulcers,

\section{Introducción}

Las prácticas más eficientes para evitar la presencia de úlceras por presión (UPP) van dirigidas a la prevención. Inician con la valoración del riesgo enfocado en la movilidad del paciente, control de esfínteres, déficit sensorial y el estado nutricional. Para la prevención y el manejo de las UPP hay que tener en cuenta que el origen de las úlceras es multifactorial, que tiene factores causales, pero también factores de riesgo con valor predictivo; además, el pronóstico de las úlceras es variable, puesto que, si tiene inmovilidad transitoria, el pronóstico depende an Experience at "Fundacion Oftalmológica of Santander" (FOSCAL). MedUNAB 2016; 19(2):115-123].

Keywords: Pressure ulcer; Practice guideline; Skin care; nursing; Risk.

\section{Resumo}

Introdução: Faz tempo, é conhecida a importância da avaliação do risco como estratégia para prevenir o desenvolvimento de úlceras de pressão (UPP), hádiretrizes de prática clínica, que se caracterizam por recolher a melhor evidencia disponível para este tipo de recomendação; a avaliação de risco e a prevenção de úlceras de pressão é um manual do Registered Nurses Association of Ontario (RNAO). As úlceras de pressãosãoum problema durante o atendimento hospitalar, porque elasafetam e prejudicam a saúde, a qualidade de vida e a funcionalida de familiar, semesquecer as implicações económicas e jurídicas decorrentes para as instituições. Objetivo: Descrever a experiencia na implementação do manual de boas práticas de enfermagem da RNAO nos serviços de hospitalização e naunidade de terapia intensiva de adultos, no período que vai de 2013-2015. Metodologia: Estudo descritivo prospectivo de aplicação do manual para a avaliação de riscos e prevenção de úlceras de pressão RNAO nos serviços de hospitalização e de unidade de terapia intensiva para adultos organizada emduas fases, a) Fase de Preparação: convenio, seleção e capacitação; b) Fase de Implementação: auditoria e monitoramento. Resultados: O uso do manual para a avaliação do risco e prevenção de úlceras de pressãofoi aplicado numapopulação de 16,264 , dos quais $6,712(41 \%)$ no ato da hospitalização, jáforam clasificados em risco de desenvolver UPP e foram submetidos a uma intervenção nassuperfícies de suporte a $5,196(77 \%)$ e um total de $250(4 \%)$ dos pacientes desenvolveram UPP estágio 2, mostrando um declínio anual e gradual da prevalência. A aceitação dos profesionais no seguimento das recomendações do manual foi de $91 \%$. Conclusões: Foi evidente naadmissão de pacientes para o serviço, um aumento no percentual de avaliação de riscos, monitoramento e reavaliação durante a hospitalização. [Esparza-Bohórquez M, Granados-Oliveros, Joya-Guevara K. Implementando do manual de boas práticas: avaliação do risco e prevenção de úlceras de pressão: experiênciana Fundação Oftalmológica do Santander (FOSCAL). MedUNAB 2016; 19 (2):115-123].

Palavras-chave: Úlcera por Pressão; Guia de Prática Clínica; Higiene da Pele; Enfermagem; Risco.

de la enfermedad aguda, pero si es una inmovilidad crónica, el pronóstico depende de la enfermedad de base (1-5).

Existen factores que influyen en la aparición de úlceras por presión como: el déficit sensorial, nutricional, alteraciones en la movilidad, exposición a la humedad y el aumento en el roce y la fricción. Es importante conocer y dar manejo a estos factores e intervenirlos. No se puede olvidar que el personal de enfermería cumple un papel muy importante en el manejo de estos usuarios y en la intervención de dichos factores causales; es por esta razón que los cuidados o 
intervenciones deben ir encaminados a prevenir y evitar la aparición de este tipo de lesiones y por consiguiente mejorar la calidad de vida de los usuarios.

Las úlceras por presión son un problema durante el proceso de atención hospitalaria porque afectan y deterioran el estado de salud, la calidad de vida y la funcionalidad familiar, sin olvidar las repercusiones económicas y legales para las instituciones que prestan servicio de salud, debido a la alta morbilidad y mortalidad que esto puede llegar a representar. Además, su presencia tiene relación directa con los procesos de atención, prevención y cuidados suministrados por el personal de enfermería; en este sentido, la literatura reporta que la presencia de UPP en las personas hospitalizadas se encuentra relacionada por el fracaso en la atención y cuidados de los pacientes $(1,2,6-8)$.

Es importante resaltar que las úlceras son originadas por presión, fricción o cizalla, o por combinación de estos tres tipos de fuerzas, afectando la piel y tejidos subyacentes; aunque la piel, la grasa y el tejido muscular pueden resistir presiones importantes por breves periodos de tiempo, la exposición prolongada a una cierta cantidad de presión ligeramente superior a la presión de llenado capilar $(32 \mathrm{~mm}$ $\mathrm{Hg}$ ) puede originar necrosis de la piel y ulceración. Tan sólo dos horas de presión ininterrumpida puede originar los cambios mencionados y su clasificación va desde el enrojecimiento de la piel (estadio I) a la presencia de lesiones con cavernas y lesiones en el músculo o hueso (estadio IV)(3).

En otro estudio descriptivo, transversal, de prevalencia cruda, se encontró que el mayor porcentaje de estadio de UPP corresponde al estadio 2 con el $30 \%$, seguido del estadio 3 con $26 \%$, el estadio 1 comprende el 13\%. En un $11 \%$ se presentaron grado 1 y 2 . El estadio 4 representa el $3 \%$. La región de localización de UPP más frecuente es la zona sacra con el $24 \%$, seguido del trocánter con el $19 \%$, los glúteos con el 11\%, el codo con el $8 \%$, maléolos y talones con el $6 \%$ cada uno (4).

En Colombia la incidencia de UPP oscila entre 7.7 y $26.9 \%$. La mayor incidencia de úlceras por presión a nivel hospitalario es en pacientes mayores de 70 años, pero se encuentra de 5 a $8 \%$ en pacientes jóvenes con lesiones medulares, lo cual constituye la causa más frecuente de retraso en la rehabilitación de estos pacientes (9).

Se encontró un estudio de corte transversal realizado en una empresa social del estado de la ciudad de Bucaramanga, el reporte de la prevalencia del evento adverso UPP que desarrollaron los usuarios hospitalizados, la prevalencia general del evento en la población de pacientes hospitalizados fue de $5.27 \%(\mathrm{n}=23$, IC $95 \%=3.37 ; 7.81)$, calculada sobre un total 436 teniendo en cuenta que cuatro usuarios presentaron más de un evento. El $31.88 \%(\mathrm{n}=139$, IC $95 \%=27.53 ; 36.48)$ presentaron factores de riesgo para desarrollar UPP según Escala de Braden (2). En este mismo estudio el estadio III fue el de mayor frecuencia $(47.82 \%)(2)$.
Diversos estudios de nivel mundial describen la incidencia de úlceras por presión en distintos países; en Canadá es del $26 \%$ en instituciones sanitarias y del $25 \%$ en centros de agudos; en Alemania se encuentra entre el 5.3\% y el 28.3\% en hospitales; en Italia es del $8.3 \%$ en hospitales; Japón del 5.1\%; Países Bajos 23.1\%. En EEUU presenta un rango entre $2.7 \%$ y $29.5 \%$; en España el estudio más amplio realizado hasta la fecha muestra una cifra en hospitales de 8.81 - 10.21 (DS) y un 8.3 en pacientes de atención domiciliaria, con una medida global por paciente de 1.97 (1).

Los estudios muestran también un incremento de los costos de la atención sanitaria en pacientes que desarrollaron úlceras por presión. Un reciente estudio europeo de costos indica que las úlceras por presión suponen entre el 1\% y el $4 \%$ de los gastos sanitarios (7). Los costos anuales del tratamiento de las úlceras por presión en Estados Unidos oscilan entre los 9.1 y los 11.6 billones de dólares, con un costo por úlcera por presión que varía entre los 21,000 y los 152,000 dólares (8).

El profesional de enfermería en el siglo XXI enfrenta el reto de mantenerse actualizado acorde con los avances de la ciencia y la tecnología, produciendo impacto en el cuidado como integrante fundamental del equipo interdisciplinario de salud. El cuidado de enfermería exige articular los conocimientos científicos, la experiencia obtenida mediante el quehacer cotidiano y el manejo de la información a través de la evidencia científica. Esto se valida con una corriente de información que empezó al final de los años 80 o principios de los 90 cuando se empezó a hablar de práctica profesional basada en la evidencia, la cual aporta seguridad y capacidad crítica consolidando los conocimientos de los cuidados brindados. En Colombia son pioneros los médicos pertenecientes a la Asociación Colombiana de Facultades de Medicina (ASCOFAME) y el Instituto Colombiano de Seguridad Social. Este último estableció un convenio con la Asociación Colombiana de Facultades de Enfermería (ACOFAEN) para realizar guías basadas en la evidencia (10).

Este avance surgió de la necesidad de responder a las preguntas y dudas de la profesión, de evolucionaren el campo de los cuidados y de reforzar la identidad como disciplina científica y como profesión (11). Los cuidados o práctica basada en la evidencia aplicada en una disciplina aumentan la calidad de los cuidados, la seguridad en los pacientes y el manejo de los riesgos en la práctica (12). Es por esta razón que se deben implementar en las instituciones de salud modelos de práctica de enfermería basada en la evidencia.

En un esfuerzo por acercar la evidencia a la práctica clínica, publica guías de buenas prácticas en enfermería, permitiendo su acceso gratuito para que las enfermeras puedan incorporarlas a su práctica diaria. Diferentes instituciones de nivel mundial son reconocidas por su compromiso con la excelencia, distinción (BPSO) otorgada 
por la RNAO en cumplimiento de los requisitos exigidos en el convenio. En Latinoamérica la Universidad de Chile y la Clínica Las Conde tienen esta distinción y en Colombia la Clínica Cardioinfantil y la Fundación Oftalmológica de Santander FOSCAL el pasado mes de abril del 2015 recibieron la distinción BPSO. Una de las guías que la FOSCAL a través del convenio con la RNAO se comprometió a implementar correspondió a la guía de valoración del riesgo y prevención de úlceras por presión. De esta manera este estudio busca describir la experiencia en la implementación de la guía de buenas prácticas de enfermería de la RNAO en dos servicios de hospitalización y en la unidad de cuidado intensivo adulto, durante un periodo de 2013 a 2015.

\section{Metodología}

La Fundación Oftalmológica de Santander FOSCAL es una institución de tercer nivel de atención y cuarto nivel de complejidad, ubicada estratégicamente en el nororiente colombiano, tiene una capacidad de 256 camas hospitalarias (229 de adultos, 19 de pediatría y 8 de obstetricia) y 46 camas de unidad de cuidado intensivo (34 para adultos y 12 pediátricas), 18 salas de cirugía, y una sala para la atención de partos; en promedio maneja un volumen de 1,584 egresos mensuales, un promedio estancia de 5.8 y un porcentaje ocupacional del $93 \%$.

Con el objeto de poder mostrar la experiencia de la implementación de la guía se generó un diseño por fases: $\square$ Fase de preparación:

Convenio, selección y capacitación: se inició con la incorporación de la guía mediante el convenio establecido por la división de enfermería de la Fundación Oftalmológica de Santander (FOSCAL) con la Asociación Nacional de Enfermeras de Ontario Canadá (RNAO) en el año 2012, en el cual se seleccionó la guía de valoración del riesgo y prevención de úlceras por presión (5), con el objetivo de brindar herramientas al profesional de enfermería para la toma de decisiones adecuadas y brindar cuidados de enfermería basados en la evidencia y de esta manera disminuir las lesiones de piel derivadas de la presión.

Se seleccionaron los servicios realizando un análisis del entorno y evaluando la prevalencia de eventos relacionados con lesiones de piel quedando como servicios pilotos para la aplicación de las recomendaciones de la guía los pisos tercero oriente y occidente de medicina interna, el quinto de neurocirugía y la unidad de cuidados intensivos adultos.

Se revisaron las recomendaciones existentes en la guía valoración del riesgo y prevención de úlceras por presión de la RNAO y se seleccionaron e implementaron en FOSCAL aquellas con mejor nivel de evidencia y las que se aplican teniendo en cuenta nuestros recursos humanos, administrativos y económicos. (Tabla 1).

La división de enfermería y la RNAO llevaron a cabo procesos relacionados con la capacitación del personal, se designaron y capacitaron líderes Champions en todas las

Tabla 1. Recomendaciones de la guía devaloración del riesgo y prevención de úlceras por presión de la Registered Nurses' Association of Ontario (RNAO).

RECOMENDACIÓN DE LA PRÁCTICA

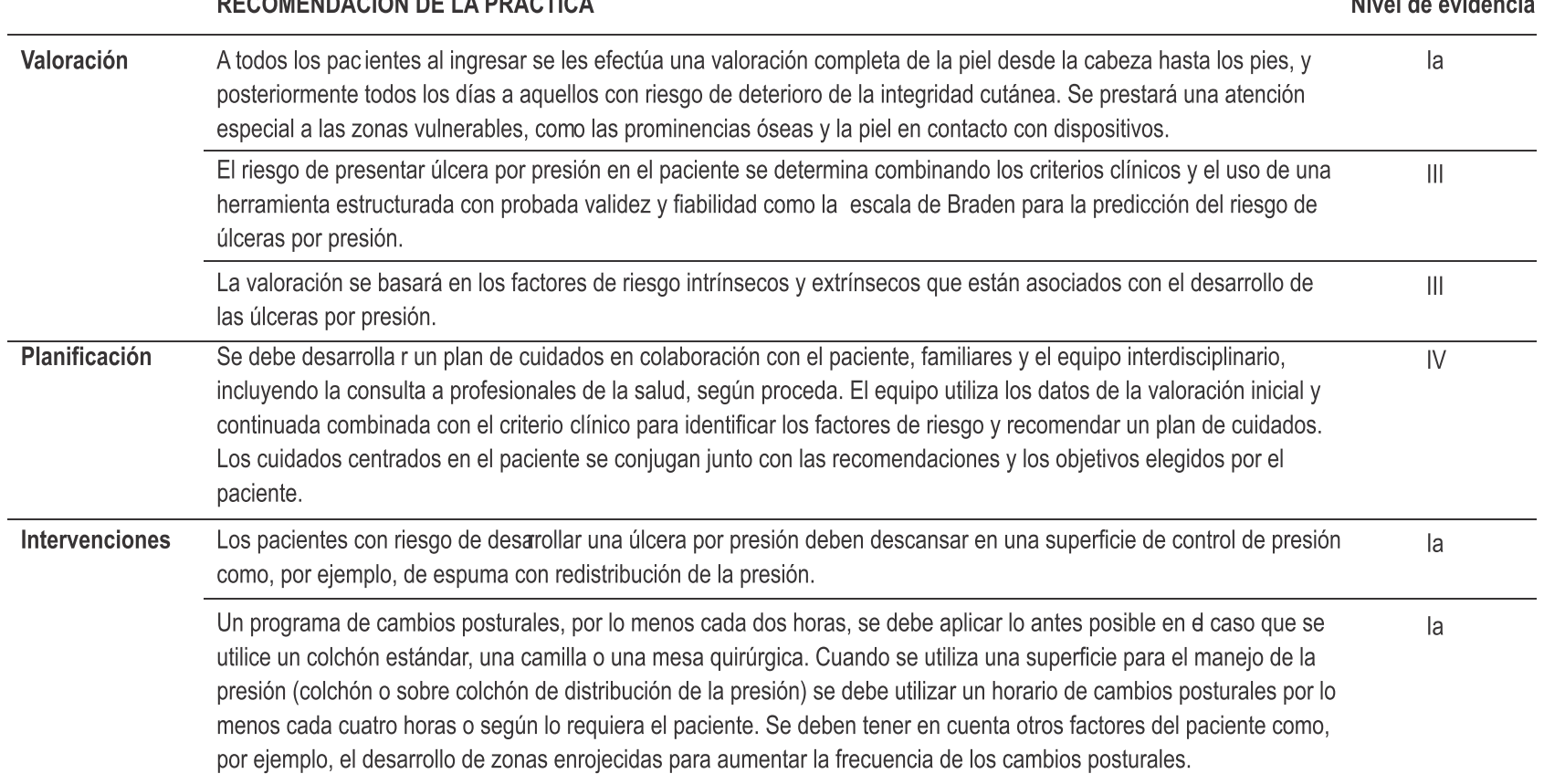




\begin{tabular}{|c|c|c|}
\hline \multirow[t]{5}{*}{ Intervenciones } & $\begin{array}{l}\text { Los talones deben estar descargados de presión en todas las posiciones. Si no es posible, las razones deben ser } \\
\text { documentadas. Los talones deben ser monitorizados y se deben aplicar estrategias de prevención. }\end{array}$ & III \\
\hline & Se debe evitar el masaje sobre las prominencias óseas y áreas enrojecidas. & IV \\
\hline & Se deben usar de forma correcta las técnicas de cambios posturales y traslados. & $\mathrm{lb}$ \\
\hline & $\begin{array}{l}\text { En el caso de personas que deban permanecer en cama: } \\
\text { - Cambios posturales cada dos horas o más frecuencia si el riesgo es elevado. } \\
\text { - Utilizar almohadas o cuñas de espuma para evitar el contacto entre las prominencias óseas. } \\
\text { - Emplear dispositivos para aliviar completamente la presión sobre los talones y las prominencias óseas de los pies. } \\
\text { - } \text { Reducir las fuerzas de cizalla manteniendo la cabecera de la cama con una elevación mínima en función de } \\
\text { - las restricciones médicas. Se recomienda una elevación de } 30^{\circ} \text { o inferior. } \\
\text { - Evitar emplear productos en forma de anillo, o aquellos que localicen la presión en otras zonas. }\end{array}$ & IV \\
\hline & $\begin{array}{l}\text { Se debe proteger la piel de la excesiva humedad y de la incontinencia para mantener la integridad de la piel: } \\
\text { - Monitorizar la ingesta de líquidos para asegurar una adecuada hidratación. } \\
\text { - Para la limpieza, utilice jabón con pH equilibrado, con agua caliente. } \\
\text { - Mantener la hidratación de la piel mediante la aplicación de agentes hidratantes, no sensibilizantes, un pH } \\
\text { - equilibrado, libre de fragancias ylo sin alcohol. } \\
\text { - Utilizar barreras protectoras tópicas para proteger la piel de la humedad. Evite aditivos y exceso de aplicación } \\
\text { - Utilizar barreras de protección (como, por ejemplo, películas líquidas de protección, hidrocoloides) o apósitos } \\
\text { de relleno para reducir las lesiones por fricción. }\end{array}$ & III \\
\hline
\end{tabular}

Fuente: Tomado de National Pressure Ulcer Advisory Panel (NPUAP).www.npuap.org/

áreas mencionadas quienes se encargaron de retroalimentar al personal de todos los servicios y realizar seguimiento inicial al proceso de implementación de la guía.

De igual manera, se seleccionaron grupos de interés (secretarias asistenciales, camilleros, personal de radiología y otros) para lograr apoyo en la metodología desde su propio rol, se identificaron las acciones que ellos deben aplicar para la implementación del plan multifactorial para evitar lesiones de piel por presión.

La escala Braden fue la herramienta seleccionada para valorar el riesgo de los usuarios. (Tabla 2).
Teniendo en cuenta la clasificación del riesgo de desarrollar úlceras por presión según el puntaje obtenido en la evaluación del riesgo con la escala de Braden, se establecieron tiempos de revaloración (Tabla 3).

\section{$\square$ Fase de implementación}

Auditoría y Seguimiento:

Se analizaron los indicadores necesarios para el seguimiento en cada servicio y se diseñaron las fichas técnicas de los indicadores por construir, se establecieron metas $\mathrm{y}$ mecanismos de seguimiento.

Los indicadores seleccionados de las guías de prevención y cuidado de la piel de la RNAO, fueron:

Tabla 2. Criterios de valoración de la escala de Braden.

\begin{tabular}{|c|c|c|c|c|}
\hline ESCALA BRADEN & & & & \\
\hline Puntos & 1 & 2 & 3 & 4 \\
\hline $\begin{array}{c}\text { Percepción } \\
\text { sensorial }\end{array}$ & $\begin{array}{l}\text { Completamente } \\
\text { limitada }\end{array}$ & Muy limitada & Levemente limitada & No alterada \\
\hline Humedad & $\begin{array}{l}\text { Completamente } \\
\text { húmeda }\end{array}$ & Muy húmeda & $\begin{array}{l}\text { Ocasionalmente } \\
\text { húmeda }\end{array}$ & Raramente húmeda \\
\hline Actividad & En cama & En silla & $\begin{array}{c}\text { Camina } \\
\text { ocasionalmente }\end{array}$ & $\begin{array}{l}\text { Camina con } \\
\text { frecuencia }\end{array}$ \\
\hline Movilidad & $\begin{array}{l}\text { Completamente } \\
\text { inmóvil }\end{array}$ & Muy limitada & ligeramente limitada & Sin limitaciones \\
\hline Nutrición & Insuficiente & $\begin{array}{l}\text { Probablemente } \\
\text { Inadecuada }\end{array}$ & Adecuada & Excelente \\
\hline $\begin{array}{c}\text { Fricción y } \\
\text { cizallamiento }\end{array}$ & Es un problema & $\begin{array}{c}\text { Es un Problema } \\
\text { potencial }\end{array}$ & $\begin{array}{c}\text { Sin problema } \\
\text { aparente }\end{array}$ & \\
\hline
\end{tabular}


Tabla 3. Tiempos de revaloración según el riesgo.

\begin{tabular}{lll}
\hline RIESGO & PUNTUACIÓN & VALORACIÓN \\
\hline Riesgo Muy Elevado & $<9$ puntos & Evaluar todos los días \\
\hline Riesgo Elevado & $10-12$ puntos & Evaluar todos los días \\
\hline Riesgo Moderado & $13-14$ puntos & Evaluar cada 2 días \\
\hline Riesgo Leve & Mayor de 15 puntos & $\begin{array}{l}\text { Evaluar cada semana o si } \\
\text { cambia el estado de salud del } \\
\text { paciente }\end{array}$ \\
\hline
\end{tabular}

Fuente: elaboración propia de los autores

\section{Indicador de valoración del riesgo:}

Número de usuarios con riesgo de desarrollar úlceras por presión

Total de usuarios ingresados

\section{Indicador de revaloración del riesgo:}

Número de usuarios con riesgo valorados según la periodicidad establecida

Total de usuarios con riesgo

\section{Indicador de intervención:}

Número de usuarios con riesgo que utilizan superficies reductoras de presión

Total de usuarios con riesgo

4.Indicador de clasificación de la úlceras por presión: Número de usuarios con riesgo que desarrollan UPP en estadios II, III y IV

Total de usuarios con riesgo

Inicialmente se diseñaron formatos físicos para la recolección de la información en cada servicio; estos contenían datos sobre fechas de valoración y revaloración, evaluación cefalocaudal, puntajes obtenidos y medidas de prevención implementadas en el paciente; estos formatos eran aplicados por la enfermera líder de la guía en la institución y por los lideres Champions designados; con los desarrollos solicitados en la historia clínica electrónica se implementó la valoración del riesgo mediante el diligenciamiento de la escala de Braden de manera electrónica, lo que permitió obtener un reporte con la totalidad de las valoraciones y revaloraciones realizadas a todos los pacientes de la institución; garantizando calidad y transparencia en el momento de obtener dicha infor-mación. Así mismo se establecieron métodos de información electrónicos y físicos con el fin de notificar al personal asistencial el riesgo del paciente de desarrollar úlceras por presión.

Para el proceso de capacitación al personal y familiares se elaboró material educativo que permitió transmitir de forma práctica las recomendaciones sobre la prevención de úlceras por presión, se diseñó cartilla de cuidado del paciente con riesgo de desarrollar lesiones de piel por presión, rotafolio con las recomendaciones de prevención de úlceras por presión, habladores con la estrategia de EVITANDO y con el resumen de las recomendaciones a seguir por el personal de enfermería.

Para el proceso de auditoría se implementaron rondas de seguimiento "protegiendo la piel FOSCAL" donde se evalúa mediante la aplicación de lista de verificación la realización de las intervenciones estratégicas seleccionadas, se mide la adherencia del personal a la guía y se recoge la información necesaria para alimentar la base de datos NursingQualityIndicatorsforReporting and Evaluation (NQUIRE®).

\section{Resultados}

A continuación, se presentan los resultados de la fase de capacitación (Tabla 4, 5,6).

Para la evaluación de la guía de valoración y prevención de úlceras por presión en la unidad de cuidados intensivos se incluyeron 4,674 pacientes admitidos. Los resultados reportaron un $48 \%$ de cumplimiento en la evaluación de los pacientes en el momento de la admisión con escala de Braden, de estos pacientes en riesgo el $90 \%$ cumplió con la revaloración a las 24 horas. El 93\% de los pacientes

Tabla 4. Fase de capacitación personal de enfermería

\begin{tabular}{ll}
\hline Año & $\begin{array}{l}\text { Personal de inducción capacitado } \\
\text { (enfermeras y auxiliares de enfermería) }\end{array}$ \\
\hline 2013 & 204 \\
\hline 2014 & 551 \\
\hline 2015 & 150 \\
\hline
\end{tabular}

Fuente: elaboración propia de los autores 
Tabla 5. Fase de capacitación grupos de interés

\begin{tabular}{|c|c|c|}
\hline \multicolumn{3}{|r|}{ GRUPOS DE INTERÉS } \\
\hline CARGO & PERSONAL & OBSERVACIONES \\
\hline & CAPACITADO & \\
\hline Secretarias & 23 & En el mes de octubre de 2015 se realizó jornada de capacitación a los \\
\hline Camilleros & 15 & grupos de apoyo identificados: camilleros, secretarias del área asistencial, \\
\hline $\begin{array}{c}\text { Tecnólogos de } \\
\text { radiología }\end{array}$ & 6 & $\begin{array}{c}\text { tecnólogos de radiología, con el fin de sensibilizarlos en su participación } \\
\text { como líderes Champions de apoyo en la implementación y sostenimiento } \\
\text { de las guías a nivel institucional. }\end{array}$ \\
\hline
\end{tabular}

Fuente: elaboración propia de los autores

Tabla 6. Fase de capacitación: socialización general de los resultados

\begin{tabular}{|c|c|c|c|}
\hline Año & Mes & $\begin{array}{l}\text { Personal } \\
\text { capacitado }\end{array}$ & Observaciones \\
\hline 2015 & JULIO & 304 & $\begin{array}{l}\text { Socialización general al personal sobre los } \\
\text { resultados obtenidos con la implementación de } \\
\text { esta iniciativa }\end{array}$ \\
\hline
\end{tabular}

Fuente: elaboración propia de los autores

descansaron sobre un colchón de alta densidad según la recomendación de la guía (Tabla 7).

Para la evaluación de la guía de valoración y prevención de úlceras por presión en los servicios de medicina interna se incluyeron 8,960 pacientes admitidos. Los resultados reportaron un $37.8 \%$ de cumplimiento en la evaluación de los pacientes en el momento de la admisión con escala de Braden, de estos pacientes en riesgo el $73.3 \%$ cumplió con la revaloración según periodicidad. El $77.6 \%$ de los pacientes descansaron sobre un colchón de alta densidad según la recomendación de la guía (Tabla 8).

Tabla 7. Valoración del riesgo y prevención de úlceras por presión en la unidad de cuidados intensivos adulto, periodo 2013 a 2015.

\begin{tabular}{lllllllll}
\hline Unidad de cuidados intensivos adulto & $\mathbf{2 0 1 3}$ & $\mathbf{\%}$ & $\mathbf{2 0 1 4}$ & $\mathbf{\%}$ & $\mathbf{2 0 1 5}$ & $\mathbf{\%}$ & Total & \% \\
\hline Total de pacientes & 1,592 & & 1,456 & & 1,626 & & 4,674 \\
\hline Usuarios valorados al ingreso & 593 & 37.2 & 559 & 38 & 1,093 & 67 & 2,245 & 48 \\
\hline Usuarios revalorados & 565 & 95.3 & 542 & 97 & 923 & 84 & 2,030 & 90 \\
\hline Intervención realizada & 580 & 97.8 & 556 & 99 & 942 & 86 & 2078 & 93 \\
\hline Usuarios con upp (estadios ii, iii, iv) & 6 & 1.0 & 12 & 2.1 & 23 & 2.1 & 41 & 1.8 \\
\hline
\end{tabular}

Fuente: elaboración propia de los autores

Tabla 8. Pacientes de los servicios de hospitalización de medicina interna en los pisos de tercero oriente y occidente con valoración y seguimiento de la guía

\begin{tabular}{lllllllll}
\hline $\begin{array}{l}\text { Total de pacientes hospitalizados por } \\
\text { año }\end{array}$ & $\begin{array}{l}\mathbf{2 0 1 3} \\
\mathrm{n=2,807}\end{array}$ & $\mathbf{2}$ & $\begin{array}{l}\mathbf{2 0 1 4} \\
\mathrm{n}=\mathbf{3 , 1 3 4}\end{array}$ & $\mathbf{2}$ & $\begin{array}{l}\mathbf{2 0 1 5} \\
\mathrm{n}=\mathbf{3 , 0 1 9}\end{array}$ & $\begin{array}{l}\text { Total } \\
\mathrm{n}=\mathbf{8 , 9 6 0}\end{array}$ \\
\hline Usuarios valorados al ingreso & 300 & 10.7 & 1,443 & 46.0 & 1,645 & 54.5 & 3,388 & 37.8 \\
\hline Usuarios revalorados & 270 & 90.0 & 1,235 & 85.6 & 908 & 69.3 & 2,413 & 73.3 \\
\hline Intervención realizada & 196 & 65.3 & 1,168 & 80.9 & 1,265 & 76.9 & 2,629 & 77.6 \\
\hline $\begin{array}{l}\text { Usuarios con Ulceras por presión UPP } \\
\text { (estadios II, III, IV) }\end{array}$ & 82 & 27.3 & 51 & 3.5 & 29 & 1.8 & 162 & 4.8 \\
\hline
\end{tabular}

Fuente: elaboración propia de los autores 
Para la evaluación de la guía de valoración y prevención de úlceras por presión en el servicio de neurocirugía se incluyeron 2,630 pacientes admitidos. Los resultados reportaron un $41 \%$ de cumplimiento en la evaluación de los pacientes en el momento de la admisión con escala de Braden, de estos pacientes en riesgo el $73.3 \%$ cumplió con la revaloración según periodicidad. El $73.1 \%$ de los pacientes descansaron sobre un colchón de alta densidad según la recomendación de la guía. (Tabla 9).

En la Figura 1 se evidencia un aumento gradual en el porcentaje de pacientes con valoración del riesgo en la admisión a la hospitalización en los tres servicios seleccionados como pilotos de la guía de valoración del riesgo y prevención de úlceras por presión.

\section{Discusión}

Durante la implementación de la guía basada en la evidencia se identificó el tener una guía de implementación como fortaleza que brinda herramientas a los líderes al tener el paso a paso garantizando el logro de los objetivos planteados de igual forma el grupo de enfermeras de la FOSCAL han respondido como líderes en la práctica con la implementación y evaluación de la guía y han trabajado por una cultura de la práctica de enfermería basada en la evidencia a nivel institucional.

El personal de enfermería ha mejorado la identificación de los usuarios con riesgo para así implementar las intervenciones establecidas como el uso de superficies de apoyo y disminuir la incidencia de úlceras de presión en la institución (11). En su revisión de literatura sobre el uso de la escala de Braden para identificar los pacientes en riesgo y el uso de superficies de apoyo como intervención en diferentes hospitales donde se evidencia la reducción de úlceras de presión al igual que en nuestra institución $(5,12-13)$.

Sumado a las estrategias anteriores la educación brindada tanto al personal de enfermería como al usuario y el cuidador principal, las cuales se consideran de gran impacto

Tabla 9. Pacientes de los servicios de hospitalización de neurocirugía servicio quinto Torre Milton Salazar con valoración y seguimiento de la guía.

\begin{tabular}{|c|c|c|c|c|c|c|c|c|}
\hline $\begin{array}{l}\text { Hospitalización neurocirugía } \\
\text { pacientes }\end{array}$ & $\begin{array}{l}2013 \\
n=785\end{array}$ & $\%$ & $\begin{array}{l}2014 \\
n=928\end{array}$ & $\%$ & $\begin{array}{l}2015 \\
n=917\end{array}$ & $\%$ & $\begin{array}{l}\text { Total } \\
n=2,630\end{array}$ & $\%$ \\
\hline Usuarios valorados al ingreso & 130 & 16.6 & 421 & 45.4 & 528 & 57.6 & 1,079 & 41 \\
\hline Usuarios revalorados & 122 & 93.8 & 345 & 82.0 & 347 & 74.0 & 814 & 73.3 \\
\hline Intervención realizada & 69 & 53.1 & 338 & 80.3 & 382 & 72.3 & 789 & 73.1 \\
\hline $\begin{array}{l}\text { Usuarios con ulceras por presión (estadios } \\
\text { II, III, IV) }\end{array}$ & 23 & 17.7 & 10 & 2.4 & 16 & 3.0 & 49 & 4.5 \\
\hline
\end{tabular}

Fuente: elaboración propia de los autores

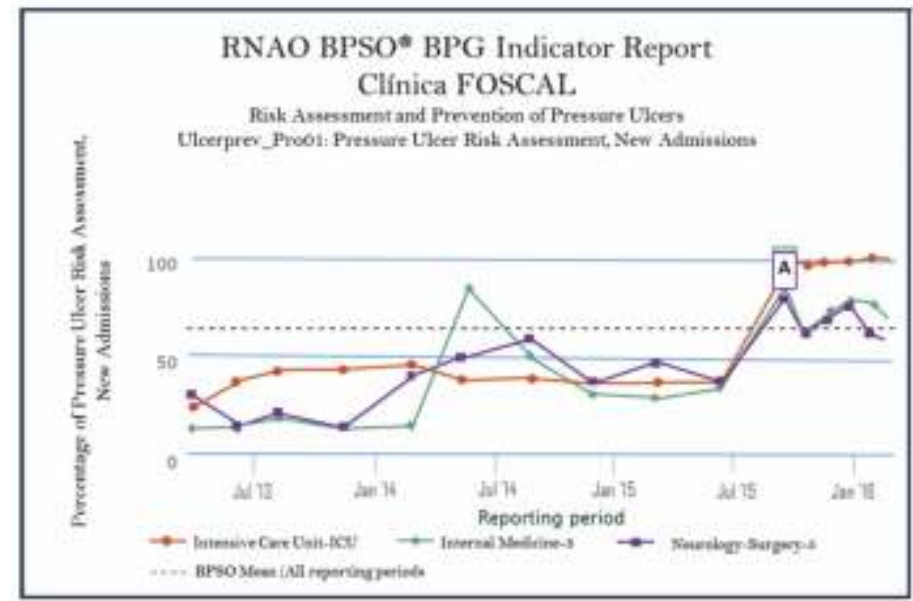

Fuente: NQUIRE, Registered Nurses Association of Ontario.

Figura 1. Porcentaje de usuarios admitidos con registro de valoración del riesgo de úlceras por presión en los servicios de unidad de cuidado intensivo, medicina interna y neurocirugía 2013 a 2015. 
en los resultados de reducción de la incidencia de úlceras por presión (14). Donde evaluaron el impacto de la implementación de intervenciones basadas en la evidencia. Otros autores documentan en su publicación donde evidencian la importancia de un programa de educación al personal de enfermería $(15,16)$.

Durante el análisis de los indicadores se identificó que un número significativo de los usuarios que ingresaban al servicio de hospitalización procedentes de urgencias presentaban lesiones de piel ocasionadas por la estancia prolongada en dicho servicio, ante esto se planteó la inclusión del servicio de urgencias como un área clave para la implementación de la guía.

Se espera realizar comparación sobre la efectividad de los cuidados para prevenir lesiones de piel recomendados por la guía de valoración del riesgo y prevención de úlceras por presión RNAO, teniendo en cuenta que se está recolectando información de las diferentes instituciones.

\section{Conclusiones}

Se estableció como cultura institucional la valoración del riesgo de los usuarios que ingresaron a la institución.

La estrategia de capacitación al personal inicia con la capacitación a un líder Champions quien se encarga de transmitir toda esta información al personal que ingresa al respectivo servicio, este líder Champions es el encargado también de realizar seguimiento a la adherencia en el cumplimiento de las recomendaciones de la guía.

Mediante la recolección de información de cada servicio en la base de datos de NQUIRE, se garantiza el registro de la evidencia científica de la institución para trabajar en pro de la transformación cultural en el tiempo.

\section{Conflicto de intereses}

Los autores manifiestan que no tienen ningún conflicto de interés.

\section{Referencias}

1. Ministerio de la Protección Social. Prevenir las úlceras por presión. Bogotá, Colombia 2009. Ministerio de la Protección Social. (Versión 1.0). Disponible en: http:// salpub.uv.es/SALPUB/practicum12/docs/guies BPC/U Iceras presion/Prevenir ulceras por presion Chile.pdf

2. Valero-CárdenasH, ParraDI, Rey-GómezR, CamargoFigueraFA. Prevalencia de úlcera por presión en una institución de salud. RevSalud UIS 2011; 43(3): 249-255. Disponible en http://www. scielo.org.co/ scielo.php?script=sci_arttext\&pid=S0121-08072011000 300005\&lng=en.
3. Palomar-Llatas F, Fornes-Pujalte B, Arantón-Areosa L, Rumbo-PrietoJM.Diferenciación de las úlceras en pacientes encamados y con enfermedades crónicas. Influencia de la humedad, fricción, cizalla y presión.EnfermDermatol2013; 7(18,19):14-25.

4. González-Consuegra RV, Cardona-Mazo DM, MurciaTrujillo PA, Matiz-Vera GD. Estudio de prevalencia de úlceras por presión en Colombia: Informe preliminar. RevFacMed 2014; 62(3): 369-77. http://dx.doi.org/ 10.15446/ revfacmed.v62n3.43004

5. Registered Nurses' Association of Ontario (2005). Risk assessment and prevention of pressure ulcers. (Revised). Toronto, Canada: Registered Nurses' Association of Ontario 2011. Disponible en: http://rnao.ca/sites/rnaoca/ files/Risk_Assessment_and_Prevention_of_Pressure_Ulc ers.pdf

6. Ministerio de Salud y Protección Social. Manual de implementación de guías de práctica clínica basadas en evidencia, en instituciones prestadoras de servicios de salud en Colombia. Bogotá, Colombia 2014. Disponible en: http://gpc.minsalud.gov.co/recursos/Documentos $\%$ 20compartidos/Manual de implementacion.pdf

7. Don R. Revis, H. Hollis Caffee. Pressuresores, surgical treatment and principles. [enlínea] 2005; Disponibleen: http://www.emedicine.com.

8. Bennett G, Bennett G, Dealey C, Posnett J. The cost of pressure ulcers in the UK. Age Ageing 2004; 33(3):230235. DOI: $10.1093 /$ ageing/afh086

9. Zulkowski K, Zulkowski K, Langemo D, Posthauer ME, the National Pressure Ulcer Advisory Panel. Coming to Consensus on Deep Tissue Injury. Adv Skin Wound Care 2005; 18(1): 28-29.

10. Ramos-De la cruz E. Enfermería basada en la evidencia.Duazary2004; 1(2): 148-152.

11. Eterovic-Díaz C, Stiepovich-Bertoni J. Enfermería basada en la evidencia y formación profesional. CiencEnferm 2010; 16(3): 9-14.Disponible en: http://www.scielo.cl/ scielo.php?script=sci arttext\&pid=S0717 95532010000300002\&lng=es.http://dx.doi.org/10.4067/S0 717-95532010000300002.

12. Urra-Medina E, Retamal-Valenzuela C, Tapia-Pinto C, Rodríguez-Vidal M. Enfermería basada en la evidencia: qué es, sus características y dilemas. Investigación y educación en enfermería 2010; 28(1): 108-118.Disponible en: http://www.redalyc.org/articulo.oa?id=105215294014. Fecha de consulta: 20 de octubre de 2016.

13. Comfort EH. Reducing Pressure Ulcer Incidence through Braden Scale Risk Assessment and Support Surface Use.Adv Skin WoundCare 2008; 21(7): 330-334.

14. Young J, Ernsting M, Kehoe A, Holmes K. Results of a clinician-led evidence-based task force initiative relating to pressure ulcer risk assessment and prevention. J WOCN 2010; 37(5): 495-503. Doi: 10.1097/WON. 0b013e3181edadcf.

15. Sinclair L, Berwiczonek H,Thurston N,Butler S,Bulloch G,Ellery C, Giesbrecht G. Evaluation of an EvidenceBased Education Program for Pressure Ulcer Prevention. J WOCN 2004; 31(1):43-50.

16. González-Consuegra RV, Pérez-Valderrama DC, Valbuena-Flor LF. Prevención de lesiones de piel: educación en el equipo de salud y familiares de personas hospitalizadas. Rev. Fac. Med. 2016; 64(2):229-38. Spanish. Doi: http://dx.doi.org/10.15446/revfacmed. v64n2.49903. 